\title{
A BOREL-CANTELLI LEMMA FOR INTERMITTENT INTERVAL MAPS
}

\author{
SÉBASTIEN GOUËZEL
}

\begin{abstract}
We consider intermittent maps $T$ of the interval, with an absolutely continuous invariant probability measure $\mu$. Kim showed that there exists a sequence of intervals $A_{n}$ such that $\sum \mu\left(A_{n}\right)=\infty$, but $\left\{A_{n}\right\}$ does not satisfy the dynamical Borel-Cantelli lemma, i.e., for almost every $x$, the set $\left\{n: T^{n}(x) \in A_{n}\right\}$ is finite. If $\sum \operatorname{Leb}\left(A_{n}\right)=\infty$, we prove that $\left\{A_{n}\right\}$ satisfies the Borel-Cantelli lemma. Our results apply in particular to some maps $T$ whose correlations are not summable.
\end{abstract}

\section{INTRODUCTION}

Let $T$ be an ergodic probability preserving transformation of a space $(X, \mu)$, and let $A_{n}$ be a sequence of subsets of $X$ with $\sum \mu\left(A_{n}\right)=+\infty$. It is an interesting question to know whether, for almost every point $x, T^{n}(x)$ belongs to $A_{n}$ infinitely often. By the classical Borel-Cantelli lemma, this holds if the sets $T^{-n} A_{n}$ are pairwise independent, but this condition is almost never satisfied for dynamical systems, so one is led to looking for weaker conditions.

If $T$ is invertible, taking $A_{n}=T^{n}(A)$ for some fixed set $A$ gives a trivial counterexample (and similar counterexamples also exist for noninvertible maps) . Hence, some regularity conditions on the sets $A_{n}$ are necessary. For uniformly hyperbolic dynamical systems, Chernov and Kleinbock have solved the problem for lots of families of balls in CK01 (see also Mau06). The partially hyperbolic case is dealt with in Dol04. Concerning non-uniformly hyperbolic (or expanding) systems, Kim has considered in Kim07 a family of interval maps with a neutral fixed points and obtained partial results. Our goal in this note is to complete these results (for the same family of maps) and obtain a full description of the situation.

Consider some parameter $\alpha>0$ and let $T_{\alpha}:(0,1] \rightarrow(0,1]$ be given by

$$
T_{\alpha}(x)= \begin{cases}x\left(1+2^{\alpha} x^{\alpha}\right) & \text { if } x \in(0,1 / 2], \\ 2 x-1 & \text { if } x \in(1 / 2,1] .\end{cases}
$$

It preserves a unique (up to multiplication by a scalar) absolutely continuous measure $\mu$, and this measure has finite mass if and only if $\alpha<1$. Henceforth, we will only consider this case, and assume that $\mu$ is normalized to be a probability measure. We will also denote by Leb the Lebesgue measure on $(0,1]$.

In Kim07, Kim proves the following result: for any $\alpha<1$, there exist intervals $A_{n}$ such that $\sum \mu\left(A_{n}\right)=\infty$ but, for almost every $x, T_{\alpha}^{n}(x) \in A_{n}$ occurs only

Date: March 9, 2007.

2000 Mathematics Subject Classification. 37A25, 37C30, 37E05.

Key words and phrases. dynamical Borel-Cantelli lemma, intermittent maps.

We thank François Maucourant for useful comments. 
finitely many times. In other words, the answer to the Borel-Cantelli problem in this setting is not always positive. On the other hand, he proves that, if $A_{n}$ is a sequence of intervals in $(d, 1]$ for some $d>0$, with $\sum \mu\left(A_{n}\right)=\infty$, and

- either $A_{n+1} \subset A_{n}$ for all $n$

- or $\alpha<(3-\sqrt{5}) / 2$

then, for almost every $x, T_{\alpha}^{n}(x)$ belongs to $A_{n}$ infinitely many times. In this note, we prove the following theorem:

Theorem 1.1. Let $\alpha<1$, and let $A_{n}$ be a sequence of intervals with $\sum \operatorname{Leb}\left(A_{n}\right)=$ $\infty$. Then, for almost every $x, T_{\alpha}^{n}(x)$ belongs to $A_{n}$ infinitely many times.

The measures $\mu$ and Leb are uniformly equivalent on every interval $(d, 1]$ (more precisely, on every interval $(d, 1]$, the density $h$ of $\mu$ with respect to Leb is Lipschitz continuous and bounded from above and below). Hence, this theorem implies the aforementioned result of Kim.

The proof involves a measurement of how sets $T_{\alpha}^{-i} A_{i}$ and $T_{\alpha}^{-j} A_{j}$ are "close to be independent". For the following informal description of the proof, assume for the sake of simplicity that the intervals $A_{n}$ are all contained in $(1 / 2,1]$. The speed of decay of correlations of the map $T_{\alpha}$ is exactly $1 / n^{\beta-1}$ for $\beta=1 / \alpha$, which means that the best estimate we could hope for is of the form

$$
\left|\mu\left(T_{\alpha}^{-i} A_{i} \cap T_{\alpha}^{-j} A_{j}\right)-\mu\left(A_{i}\right) \mu\left(A_{j}\right)\right| \leq \frac{C \mu\left(A_{j}\right)}{(j-i)^{\beta-1}},
$$

for $j>i$. This estimate indeed holds, and implies Theorem 1.1 when the sequence $1 / n^{\beta-1}$ is summable, that is, when $\alpha<1 / 2$. However, it is not sufficient when $1 / 2 \leq$ $\alpha<1$, and we need to know further terms in the asymptotics of the correlations. Here comes into play our main technical tool, the renewal sequence of transfer operators, studied by Sarig in Sar02. Using the results in Gou04a, we will prove the existence of a sequence $c_{n}$ converging to 1 such that

$$
\left|\mu\left(T_{\alpha}^{-i} A_{i} \cap T_{\alpha}^{-j} A_{j}\right)-c_{j-i} \mu\left(A_{i}\right) \mu\left(A_{j}\right)\right| \leq \frac{C \mu\left(A_{j}\right)}{(j-i)^{\beta}} .
$$

This sequence is of the form $c_{n}=1+c / n^{\beta-1}+o\left(1 / n^{\beta-1}\right)$ for some nonzero constant $c$, which shows that (1.2) is indeed optimal. For the purposes of the Borel-Cantelli problem, (1.3) is sufficient and will imply Theorem 1.1 in all cases, since the sequence $1 / n^{\beta}$ is summable whenever $\alpha<1$.

On the technical level, the results in [Sar02, Gou04a] deal with spaces of Lipschitz functions. However, the essential results are formulated in an abstract Banach spaces framework. They can therefore also be applied to spaces of functions with bounded variation, which is what is needed here to deal with the characteristic functions of intervals.

Remark 1.2. Theorem 1.1 still holds for transformations with an even more neutral fixed point, as soon as there is still an absolutely continuous invariant probability measure. This is for example the case if the fixed point is of the form $x+x^{2}(\log x)^{2}$, or more generally for the class of maps introduced by Holland in $\mathrm{Hol05}$. However, the results of Gou04a are not sufficient to prove this, and one needs to use results in the unpublished thesis Gou04b, for example Remark 2.4.8 or Remark 2.4.11. 


\section{Abstract tools}

First of all, let us recall a criterion implying the Borel-Cantelli property (proved e.g. in Spi64, Proposition 6.26.3]):

Theorem 2.1. Let $B_{n}$ be sets of a probability space $(X, \mu)$ with $\sum \mu\left(B_{n}\right)=\infty$. Assume that

$$
\limsup _{n \rightarrow \infty} \frac{\sum_{0 \leq i<j<n} \mu\left(B_{i} \cap B_{j}\right)}{\left(\sum_{j=0}^{n-1} \mu\left(B_{j}\right)\right)^{2}} \leq \frac{1}{2} .
$$

Then almost every point of $X$ belongs to infinitely many $B_{n}$ 's.

We will apply this result to $B_{n}=T_{\alpha}^{-n}\left(A_{n}\right)$. Hence, we need a good quantitative estimate on $\mu\left(T_{\alpha}^{-i} A_{i} \cap T_{\alpha}^{-j} A_{j}\right)$. This estimate will be provided by renewal sequences of transfer operators, as used by Sarig in Sar02. For our purpose, the following abstract result will be most useful. Let $\overline{\mathbb{D}}$ be the closed unit disk in $\mathbb{C}$.

Theorem 2.2. Let $\mathcal{B V}$ be a Banach space, and let $\left(R_{n}\right)_{n \geq 1}$ be a sequence of continuous linear operators on $\mathcal{B V}$. Assume that, for some $\beta>1, \sum_{k>n}\left\|R_{k}\right\|=O\left(1 / n^{\beta}\right)$. Hence, $R(z)=\sum R_{n} z^{n}$ and $R^{\prime}(z)=\sum n R_{n} z^{n-1}$ are well defined operators on $\mathcal{B V}$, for $z \in \overline{\mathbb{D}}$. Assume moreover that 1 is a simple isolated eigenvalue of $R(1)$, and that the corresponding eigenprojector $P$ satisfies $P R^{\prime}(1) P=\gamma P$ for some $\gamma \neq 0$. Assume also that, for any $z \in \overline{\mathbb{D}} \backslash\{1\}, I-R(z)$ is invertible on $\mathcal{B V}$.

Let $T_{n}=\sum_{l=1}^{\infty} \sum_{k_{1}+\cdots+k_{l}=n} R_{k_{1}} \ldots R_{k_{l}}$. This operator acts continuously on $\mathcal{B V}$. Then there exists a sequence $c_{n} \in \mathbb{C}$ converging to 1 such that $T_{n}-c_{n} P / \gamma=$ $O\left(1 / n^{\beta}\right)$.

Proof. Gou04a, Theorem 5.4] (for large enough $N$ ) shows that $T_{n}$ converges to $P / \gamma$, and that there exists a sequence of operators $Q_{n}$ such that $T_{n}-P Q_{n} P=O\left(1 / n^{\beta}\right)$. This theorem even gives a closed form expression for $Q_{n}$, but we will not need it.

Since $P$ is a one-dimensional projection, there exists a complex number $d_{n}$ such that $P Q_{n} P=d_{n} P$. The convergence of $T_{n}$ to $P / \gamma$ shows that $d_{n}$ converges to $1 / \gamma$. We obtain the theorem for $c_{n}=\gamma d_{n}$.

In Sar02, Gou04a, this theorem is applied by taking $R_{n}$ to be the "first return transfer operators" to $Y=(1 / 2,1]$, acting on the space of Lipschitz continuous functions on $Y$. Here, we will use the same operators $R_{n}$, but we will use for $\mathcal{B V}$ the space of functions of bounded variation on $Y$.

\section{Proof of the Main theorem}

In all this section, we fix $\alpha \in(0,1)$ and write $T$ for $T_{\alpha}$. Let also $\beta=1 / \alpha$.

Let $Y=(1 / 2,1]$, let $\varphi: Y \rightarrow \mathbb{N}^{*}$ denote the first return time from $Y$ to itself. Let also $\widehat{T}$ be the transfer operator associated to $T$, given for $f \in L^{1}$ (Leb) by

$$
\widehat{T} f(x)=\sum_{T y=x} f(y) / T^{\prime}(y) .
$$

Let $R_{n} f=\widehat{T}^{n}\left(1_{Y \cap\{\varphi=n\}} f\right)$, and $T_{n} f=1_{Y} \widehat{T}^{n}\left(1_{Y} f\right)$. These operators act on $L^{1}(Y)$. Moreover, $R_{n}$ corresponds to considering the first returns at time $n$, while $T_{n}$ 
considers all returns at time $n$. It is therefore easy to check the following renewal equation (see e.g. [Sar02, Proposition 1]):

$$
T_{n}=\sum_{l=1}^{\infty} \sum_{k_{1}+\cdots+k_{l}=n} R_{k_{1}} \ldots R_{k_{l}} .
$$

Let $\mathcal{B V}$ be the space of functions of bounded variation on $Y$. An element $f$ of $\mathcal{B V}$ is a bounded function on $\mathbb{R}$, supported in $Y$, and its norm is

$$
\operatorname{Var}(f):=\sup _{N \in \mathbb{N}} \sup _{x_{0}<\cdots<x_{N}} \sum_{i=0}^{N-1}\left|f\left(x_{i+1}\right)-f\left(x_{i}\right)\right|,
$$

where the $x_{i}$ 's are real numbers (not necessarily in $Y$ ). In particular, $\|f\|_{L^{\infty}} \leq$ $\operatorname{Var}(f) / 2$.

Lemma 3.1. The operators $R_{n}$ acting on $\mathcal{B V}$ satisfy the assumptions of Theorem 2.2. The spectral projection $P$ corresponding to the eigenvalue 1 of $R(1)$ is given by

$$
P f=\frac{\left(\int_{Y} f \mathrm{dLeb}\right)}{\mu(Y)} h_{Y}
$$

where $h_{Y}$ is the restriction to $Y$ of the density $h$ of the invariant probability measure $\mu$. Additionally, $P R^{\prime}(1) P=P / \mu(Y)$.

Proof. This lemma is proved in Gou04a for the action of $R_{n}$ on the space $\mathcal{L}$ of Lipschitz functions on $Y$. We will adapt this proof to the space $\mathcal{B V}$.

The set $\{\varphi=n\}$ is a subinterval $I_{n}$ of $Y$, and $T^{n}$ is a diffeomorphism between $I_{n}$ and $Y$. Moreover, $\left|I_{n}\right| \sim c / n^{\beta+1}$ for some constant $c>0$, and the distortion of $T^{n}$ on $I_{n}$ is uniformly bounded, independently of $n$, in the following sense: there exists $C>0$ such that, for all $x, y \in I_{n}$,

$$
\left|1-\frac{\left(T^{n}\right)^{\prime}(x)}{\left(T^{n}\right)^{\prime}(y)}\right| \leq C\left|T^{n} x-T^{n} y\right|
$$

See e.g. You99, Section 6] for a proof of these facts. Let $\psi_{n}: Y \rightarrow I_{n}$ be the inverse of $T^{n}$ on $I_{n}$, so that

$$
R_{n} f(x)=\psi_{n}^{\prime}(x) f\left(\psi_{n} x\right)
$$

Then

$$
\operatorname{Var}\left(R_{n} f\right) \leq\left\|\psi_{n}^{\prime}\right\|_{L^{\infty}} \operatorname{Var}\left(f \circ \psi_{n}\right)+\|f\|_{L^{\infty}} \operatorname{Var}\left(\psi_{n}^{\prime}\right) \leq C\left|I_{n}\right| \operatorname{Var}(f) .
$$

In particular,

$$
\left\|R_{n}\right\|_{\mathcal{B V} \rightarrow \mathcal{B V}} \leq \frac{C}{n^{\beta+1}}
$$

As in Theorem 2.2, we define for $z \in \overline{\mathbb{D}}$ an operator $R(z)=\sum R_{n} z^{n}$. By (3.8), this operator is well defined on $\mathcal{B V}$. Moreover, by Gou04a, Paragraph 6.3], $R(z)$ also acts continuously on the space $\mathcal{L}$ of Lipschitz continuous functions on $Y$, and satisfies the following properties. First of all, $R(z)$ satisfies a Lasota-Yorke inequality between $\mathcal{L}$ and $L^{1}$. Hence, by the theorem of Ionescu-Tulcea and Marinescu, any eigenfunction of $R(z)$ (for an eigenvalue of modulus 1 ) which belongs to $L^{1}$ belongs in fact to $\mathcal{L}$. Moreover, for $z \in \overline{\mathbb{D}} \backslash\{1\}, I-R(z)$ is invertible on $\mathcal{L}$, while $R(1)$ has a simple eigenvalue at 1 , the corresponding eigenfunction being $h_{Y}$

Let us now prove that, for any $z \in \overline{\mathbb{D}}$, the essential spectral radius of $R(z)$ acting on $\mathcal{B V}$ is $<1$. This could be proved by mimicking the arguments in Ryc83, but 
it is easier to refer to Rue96, Theorem B.1]. Indeed, this theorem shows that the essential spectral radius of $R(z)$ is bounded by $\left\|z^{\varphi} /\left(T^{\varphi}\right)^{\prime}\right\|_{L^{\infty}}<1$.

Let $z \in \overline{\mathbb{D}} \backslash\{1\}$. If $I-R(z)$ were not invertible on $\mathcal{B V}$, then there would exist a function $f \in \mathcal{B V}$ such that $R(z) f=f$. The function $f$ would in particular belong to $L^{1}$, hence, by the above argument, it would belong to $\mathcal{L}$. This is a contradiction since $I-R(z)$ is invertible on $\mathcal{L}$. In the same way, we check that $R(1)$ has a simple eigenvalue at 1 , the eigenfunction still being the density of the invariant measure. Moreover, the eigenprojection is given by (3.4).

We compute finally $P R^{\prime}(1) P$. The formula for $P f$ gives

$$
P R^{\prime}(1) P f=\frac{\left(\int_{Y} R^{\prime}(1) h_{Y} \mathrm{dLeb}\right)}{\mu(Y)} \frac{\left(\int_{Y} f \mathrm{dLeb}\right)}{\mu(Y)} h_{Y}=\gamma P f
$$

for $\gamma=\left(\int_{Y} R^{\prime}(1) h_{Y} \mathrm{dLeb}\right) / \mu(Y)$. Moreover,

$$
\int R_{n} h_{Y} \mathrm{dLeb}=\int \widehat{T}^{n}\left(1_{\{\varphi=n\}} h_{Y}\right) \mathrm{dLeb}=\int 1_{\{\varphi=n\}} h_{Y} \mathrm{dLeb}=\mu\{\varphi=n\} .
$$

Summing these formulas over $n$ gives

$$
\int R^{\prime}(1) h_{Y} \mathrm{dLeb}=\sum n \mu\{\varphi=n\}=\int_{Y} \varphi \mathrm{d} \mu=1
$$

by Kac Formula. Hence, $\gamma=1 / \mu(Y)$.

Corollary 3.2. There exist $C>0$, and a sequence $c_{n}$ of complex numbers converging to 1 when $n$ tends to infinity, such that, for any functions $f, g$ supported in $Y$, for any $n>0$,

$$
\left|\int f \cdot g \circ T^{n} \mathrm{dLeb}-c_{n}\left(\int f \mathrm{dLeb}\right)\left(\int g \mathrm{~d} \mu\right)\right| \leq \frac{C\|f\|_{\mathcal{B V}}\|g\|_{L^{1}(\mathrm{Leb})}}{n^{\beta}} .
$$

Proof. We have

$$
\int f \cdot g \circ T^{n} \mathrm{dLeb}=\int 1_{Y} \widehat{T}^{n}\left(1_{Y} f\right) g \mathrm{dLeb}=\int T_{n} f \cdot g \mathrm{dLeb} .
$$

Moreover, by (3.2), Lemma 3.1 and Theorem 2.2 there exist a sequence $c_{n}$ converging to 1 and a constant $C$ such that

$$
\begin{aligned}
\left\|T_{n} f-c_{n}\left(\int_{Y} f \mathrm{dLeb}\right) h_{Y}\right\|_{\mathcal{B V}} & =\left\|T_{n} f-c_{n} \mu(Y) P f\right\|_{\mathcal{B V}} \leq\|f\|_{\mathcal{B} \mathcal{V}}\left\|T_{n}-c_{n} \mu(Y) P\right\| \\
& \leq \frac{C\|f\|_{\mathcal{B V}}}{n^{\beta}} .
\end{aligned}
$$

Together with (3.13), this concludes the proof.

Proof of Theorem 1.1. Let first $A_{n}$ be a sequence of intervals contained in $(1 / 2,1]$, with $\sum \operatorname{Leb}\left(A_{n}\right)=\infty$ (or, equivalently, $\sum \mu\left(A_{n}\right)=\infty$ ). Let $B_{n}=T^{-n} A_{n}$. Let $j>i$. Applying Corollary 3.2 to $f=1_{A_{i}} h_{Y}, g=1_{A_{j}}$ and $n=j-i$, we get

$$
\begin{aligned}
& \left|\mu\left(B_{i} \cap B_{j}\right)-c_{j-i} \mu\left(B_{i}\right) \mu\left(B_{j}\right)\right| \\
& =\left|\int 1_{A_{i}} h_{Y} \cdot 1_{A_{j}} \circ T^{j-i} \mathrm{dLeb}-c_{j-i}\left(\int 1_{A_{i}} h_{Y} \mathrm{dLeb}\right)\left(\int 1_{A_{j}} \mathrm{~d} \mu\right)\right| \\
& \leq \frac{C \operatorname{Var}\left(1_{A_{i}} h_{Y}\right) \operatorname{Leb}\left(A_{j}\right)}{(j-i)^{\beta}} .
\end{aligned}
$$


The function $h$ is Lipschitz continuous on $Y$, and bounded from below. In particular, $\operatorname{Leb}\left(A_{j}\right) \leq C \mu\left(A_{j}\right)=C \mu\left(B_{j}\right)$. We conclude

$$
\left|\mu\left(B_{i} \cap B_{j}\right)-c_{j-i} \mu\left(B_{i}\right) \mu\left(B_{j}\right)\right| \leq \frac{C \mu\left(B_{j}\right)}{(j-i)^{\beta}} .
$$

Let $\varepsilon>0$. Let $K$ be such that, for $n \geq K,\left|c_{n}\right| \leq 1+\varepsilon$. Then

$$
\begin{aligned}
\sum_{0 \leq i<j<n} \mu\left(B_{i} \cap B_{j}\right) \leq \sum_{0 \leq i<j<n}\left|c_{j-i}\right| \mu\left(B_{i}\right) \mu\left(B_{j}\right)+\sum_{j=1}^{n-1}\left(\sum_{i=0}^{j-1} \frac{C^{\prime}}{(j-i)^{\beta}}\right) \mu\left(B_{j}\right) \\
\leq \sum_{0 \leq i<j<n}(1+\varepsilon) \mu\left(B_{i}\right) \mu\left(B_{j}\right)+\sum_{j=1}^{n-1}\left(K \sup _{p \in \mathbb{N}}\left|c_{p}\right|+\sum_{p=1}^{\infty} \frac{C^{\prime}}{p^{\beta}}\right) \mu\left(B_{j}\right) .
\end{aligned}
$$

Therefore,

$$
\frac{\sum_{0 \leq i<j<n} \mu\left(B_{i} \cap B_{j}\right)}{\left(\sum_{j=0}^{n-1} \mu\left(B_{j}\right)\right)^{2}} \leq \frac{1+\varepsilon}{2}+\left(K \sup _{p \in \mathbb{N}}\left|c_{p}\right|+\sum_{p=1}^{\infty} \frac{C^{\prime}}{p^{\beta}}\right) \frac{1}{\sum_{j=0}^{n-1} \mu\left(B_{j}\right)} .
$$

Since $\sum_{j \in \mathbb{N}} \mu\left(B_{j}\right)=\infty$, this upper bound is at most $1 / 2+\varepsilon$ for large enough $n$. We have proved that

$$
\limsup _{n \rightarrow \infty} \frac{\sum_{0 \leq i<j<n} \mu\left(B_{i} \cap B_{j}\right)}{\left(\sum_{j=0}^{n-1} \mu\left(B_{j}\right)\right)^{2}} \leq \frac{1}{2} .
$$

By Theorem 2.1 this concludes the proof in this case.

Consider now $A_{n}$ an arbitrary sequence of intervals in $(0,1]$ with $\sum \operatorname{Leb}\left(A_{n}\right)=$ $\infty$. Let $A_{n}^{\prime}=T^{-1}\left(A_{n+1}\right) \cap(1 / 2,1]$. Since $\operatorname{Leb}\left(A_{n}^{\prime}\right)=\operatorname{Leb}\left(A_{n}\right) / 2$, this sequence of intervals satisfies $\sum \operatorname{Leb}\left(A_{n}^{\prime}\right)=\infty$, and $A_{n}^{\prime}$ is a subinterval of $(1 / 2,1]$. The first part of the proof shows that, for almost every $x, T^{n} x$ belongs to $A_{n}^{\prime}$ infinitely often. However, if $T^{n}(x) \in A_{n}^{\prime}$, then $T^{n+1}(x) \in A_{n+1}$. This concludes the proof.

\section{REFERENCES}

[CK01] Nikolai Chernov and Dmitry Kleinbock. Dynamical Borel-Cantelli lemmas for Gibbs measures. Israel J. Math., 122:1-27, 2001.

[Dol04] Dmitry Dolgopyat. Limit theorems for partially hyperbolic systems. Trans. Amer. Math. Soc., 356:1637-1689, 2004.

[Gou04a] Sébastien Gouëzel. Sharp polynomial estimates for the decay of correlations. Israel J. Math., 139:29-65, 2004.

[Gou04b] Sébastien Gouëzel. Vitesse de décorrélation et théorèmes limites pour les applications non uniformément dilatantes. PhD thesis, Université Paris Sud, 2004.

[Hol05] Mark Holland. Slowly mixing systems and intermittency maps. Ergodic Theory Dynam. Systems, 25:133-159, 2005.

[Kim07] Dong Han Kim. the dynamical Borel-Cantelli lemma for interval maps. Discrete Contin. Dyn. Syst., 17:891-900, 2007.

[Mau06] François Maucourant. Dynamical Borel-Cantelli lemma for hyperbolic spaces. Israel J. Math., 152:143-155, 2006.

[Rue96] David Ruelle. Functional equation for dynamical zeta functions of Milnor-Thurston type. Communications in Mathematical Physics, 88:63-88, 1996.

[Ryc83] Marek Rychlik. Bounded variation and invariant measures. Studia Math., 76(1):69-80, 1983.

[Sar02] Omri Sarig. Subexponential decay of correlations. Invent. Math., 150:629-653, 2002. 
[Spi64] Frank Spitzer. Principles of random walk. The University Series in Higher Mathematics. D. Van Nostrand Co., Inc., Princeton, N.J.-Toronto-London, 1964.

[You99] Lai-Sang Young. Recurrence times and rates of mixing. Israel J. Math., 110:153-188, 1999.

IRMaR, Université de Rennes 1, Campus de Beaulieu, BÂtiment 22, 35042 Rennes Cedex, France.

E-mail address: sebastien.gouezel@univ-rennes1.fr 